\title{
Nuclear receptor corepressors partner with class II histone deacetylases in a Sin3-independent repression pathway
}

\author{
Eric Y. Huang, ${ }^{1,3}$ Jinsong Zhang, ${ }^{1,3}$ Eric A. Miska, ${ }^{2}$ Matthew G. Guenther, ${ }^{1}$ Tony Kouzarides, ${ }^{2}$ \\ and Mitchell A. Lazar ${ }^{1,4}$ \\ ${ }^{1}$ Division of Endocrinology, Diabetes, and Metabolism, Departments of Medicine and Genetics, and The Penn Diabetes \\ Center, University of Pennsylvania School of Medicine, Philadelphia, Pennsylvania 19104 USA; $^{2}$ The Wellcome/Cancer \\ Research Campaign (CRC) Institute, CB21QR Cambridge UK
}

Transcriptional repression mediated by corepressors N-CoR and SMRT is a critical function of nuclear hormone receptors, and is dysregulated in human myeloid leukemias. At the present time, these corepressors are thought to act exclusively through an mSin3/HDAC1 complex. Surprisingly, however, numerous biochemical studies have not detected N-CoR or SMRT in mSin3- and HDAC1-containing complexes. Each corepressor contains multiple repression domains (RDs), the significance of which is unknown. Here we show that these RDs are nonredundant, and that one RD, which is conserved in N-CoR and SMRT, represses transcription by interacting directly with class II HDAC4 and HDAC5. Endogenous N-CoR and SMRT each associate with HDAC4 in a complex that does not contain mSin3A or HDAC1. This is the first example of a single corepressor utilizing distinct domains to engage multiple HDAC complexes. The alternative HDAC complexes may mediate specific repression pathways in normal as well as leukemic cells.

[Key Words: mSin3; HDAC1; HDAC4; HDAC5; chromatin; nuclear receptors; N-CoR; SMRT]

Received November 3, 1999; revised version accepted November 23, 1999.

Recently, there has been intense interest in targeted acetylation and deacetylation of proteins, including histones, as a mechanism regulating eukaryotic gene expression (for reviews, see Struhl 1998; Kouzarides 1999). Histone acetyltransferases (HATs) act as cofactors for sequence-specific DNA-binding proteins that function as activators of transcription. Hyperacetylation of histones and other proteins increases the accessibility of chromatin templates, thereby enhancing transcription of genes to which the HATs have been targeted. Different HATs function as coactivators with distinct classes of activators (Korzus et al. 1998). Some HATs bind directly to activators, whereas others are recruited indirectly as part of multiprotein complexes. For nuclear hormone receptors (NRs), key coactivators include the p160 class (for review, see Glass et al. 1997), p300/CBP-associated factor (PCAF) (Blanco et al. 1998), and CREB-binding protein (CBP) (Chakravarti et al. 1996; Hanstein et al. 1996; Kamei et al. 1996). All of these are HATs that interact directly with the NRs in the presence of ligand. CBP is a coactivator for nearly all classes of activators, whereas the p160 coactivators function more specifically with NRs. Consistent with this, CBP can be recruited indirectly to NRs via the p160 coactivators (Westin et al. 1998).

\footnotetext{
${ }^{3}$ These authors contributed equally to this work.

${ }^{4}$ Corresponding author.

E-MAIL lazar@mail.med.upenn.edu; FAX (215) 898-5408.
}

Transcriptional repression is also an important component of eukaryotic gene regulation. From an enzymatic standpoint, repression is the converse of activation; recruitment of histone deacetylases (HDACs) leads to a more compact, repressive state of chromatin (for review, see Pazin and Kadonaga 1997). Whereas the coactivators often have intrinsic HAT activity, corepressors recruit separate molecules with HDAC activity. Two known yeast HDACs, Hda1 and Rpd3, are found in distinct complexes (Rundlett et al. 1996). Mammalian HDACs fall into two classes. Class I HDAC1, HDAC2, and HDAC3 are highly related to yeast $\mathrm{Rpd} 3$, whereas class II HDAC4, HDAC5, and HDAC6 more closely resemble the yeast protein Hda1 (Fischle et al. 1999; Grozinger et al. 1999; Miska et al. 1999; Verdel and Khochbin 1999).

Nuclear receptor corepressor (N-CoR) (Horlein et al. 1995) and silencing mediator for retinoid and thyroid receptors (SMRT) (Chen and Evans 1995) function as corepressors not only for nuclear hormone receptors, but for multiple classes of transcription factors including homeodomain and POZ domain-containing proteins, such as Pit-1 (Xu et al. 1998) and PLZF (Hong et al. 1997; Grignani et al. 1998; He et al. 1998; Lin et al. 1998). N-CoR/SMRT-mediated repression pathways play pathophysiological roles in acute promyelocytic leukemia (APL) (Grignani et al. 1998; He et al. 1998; Lin et al. 1998) and acute myelogenous leukemia (AML) (Gelmetti et al. 1998; Lutterbach et al. 1998; Wang et al. 1998) as 
well as thyroid hormone resistance (Safer et al. 1997; Yoh et al. 1997). N-CoR and SMRT are large proteins (each $\sim 270 \mathrm{kD}$ ) containing multiple highly conserved functional domains (Horlein et al. 1995; Ordentlich et al. 1999; Park et al. 1999). The carboxyl terminus is responsible for interaction with unliganded NRs /Chen and Evans 1995; Horlein et al. 1995; Seol et al. 1996; Zamir et al. 1996), whereas the amino terminus mediates active transcriptional repression. The amino terminus contains multiple, separable, nonredundant repression domains (RDs) (Horlein et al. 1995; Ordentlich et al. 1999; Park et al. 1999). N-CoR and SMRT interact indirectly with HDAC1 by associating with mSin3 (Alland et al. 1997; Heinzel et al. 1997; Nagy et al. 1997), the mammalian homolog of yeast Sin3 that was first identified as a corepressor for Mad/Max (Ayer et al. 1995). Both mammalian and yeast Sin 3 repress transcription by associating with class I HDACs (Hassig et al. 1997).

A number of observations led us to postulate the existence of $\mathrm{mSin} 3 / \mathrm{HDAC} 1$-independent repression mechanisms. Biochemical characterization of $\mathrm{mSin} 3$ and HDAC complexes has confirmed their mutual association but, surprisingly, has not revealed an association with endogenous N-CoR or SMRT (Hassig et al. 1997; Tong et al. 1998; Wade et al. 1998; Zhang et al. 1998). Furthermore, although highly conserved between $\mathrm{N}-\mathrm{CoR}$ and SMRT, the multiple RDs within a given corepressor have very different sequences. We found that the RDs do not all compete for the same limiting factors and that all RDs do not interact with mSin3A. RD3, which does not interact with $\mathrm{mSin} 3 \mathrm{~A}$, interacts directly with class II HDAC4 and HDAC5. This interaction correlates with the repressor activity of the RD3 polypeptide. Endogenous N-CoR and SMRT both interact strongly with HDAC4 in a complex that does not contain mSin3A or HDAC1. Thus, different RDs in nuclear receptor corepressors function to recruit multiple, distinct HDAC complexes.

\section{Results}

$N$-CoR immunoprecipitates possess histone deacetylase activity in the absence of $\mathrm{mSin} 3 \mathrm{~A}$ and HDAC1

We are interested in the mechanisms of repression by $\mathrm{N}$-CoR and SMRT. We have confirmed the in vitro
mSin3 interaction with nuclear receptor CoRs using recombinant proteins as has been reported by others (references in Alland et al. 1997; Heinzel et al. 1997; data not shown; Fig. 2e, below). In addition, HDAC activity was associated with endogenous N-CoR in 293T (Fig. 1a) and HeLa cells (data not shown). However, although we were easily able to detect interactions between endogenous mSin3A and HDACl in 293T and HeLa cells, the presence of N-CoR was not detected in these complexes in either cell line (Fig. 1b; data not shown). This is consistent with the failure of others to identify N-CoR or SMRT in mSin 3 and HDAC1 complexes. Furthermore, neither mSin3 nor HDAC1 coimmunoprecipitated with endogenous N-CoR (Fig. 1b).

\section{Nuclear receptor corepressors contain an mSin3-independent repression domain}

We have confirmed the presence of multiple RDs in NCoR and SMRT (Horlein et al. 1995; Ordentlich et al. 1999; Park et al. 1999), each of which represses transcription when fused to the Gal4 DNA-binding domain (DBD) (summarized in Fig. 2a). Overexpression of an N-CoR RD1 polypeptide fused to a nuclear localization signal and HA epitope tag (NLS-HA-RD1) blocked repression of a Gal4-responsive reporter gene by Gal4-N-CoR RD1, suggesting that limiting factors (potentially including mSin3) mediated RD1 function (Fig. 2b). NLS-HASMRT RD3 was expressed at similar levels (Fig. 2c) and also localized to the nucleus (Fig. 2d) but, unlike NLSHA-RD1, had little effect on repression by Gal4-RD1 (Fig. 2b). This suggests that RD3 functioned by interacting with a factor distinct from the critical components of the RD1 repression mechanism. In agreement with published reports (Alland et al. 1997; Heinzel et al. 1997), RD1 from N-CoR as well as SMRT interacted with mSin3A (Fig. 2e). In contrast, we detected little or no interaction between $\mathrm{mSin} 3 \mathrm{~A}$ and $\mathrm{RD} 3$ polypeptides from either N-CoR or SMRT (Fig. 2e). Nevertheless, histone deacetylation is likely to be a key effector of the RD3 repression mechanism, because repression by SMRT RD3 was inhibited by the HDAC inhibitor trichostatin A (TSA) (Fig. 2f).

\section{$N$-CoR associates with a class II HDAC}

The lack of a strong association between N-CoR and
Figure 1. Endogenous N-CoR immunoprecipitates deacetylase activity without coprecipitating HDAC1. (a) Whole-cell 293T extracts were prepared and immunoprecipitated with either control IgG or anti-N-CoR IgG. Immunoprecipitates were assayed directly for histone deacetylase activity and are displayed as counts per minute of ${ }^{3} \mathrm{H}$-labeled acetic acid released. S.E.M. is shown. (b) 293T cell extracts were immunoprecipitated with IgG, anti-N-CoR, anti-mSin3A, or anti-HDAC1, separated by SDS-PAGE, and immunoblotted for the presence of $\mathrm{N}-\mathrm{CoR}, \mathrm{mSin} 3 \mathrm{~A}$, and HDAC1. a

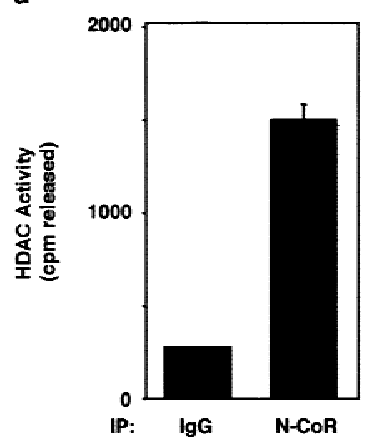

b

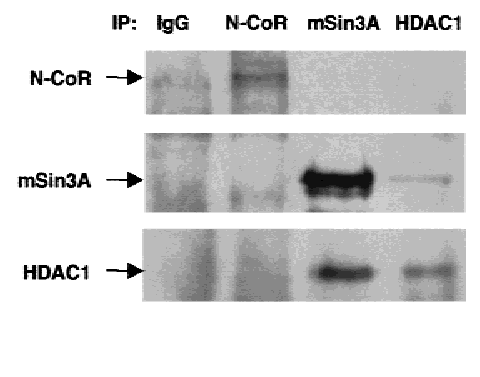


a

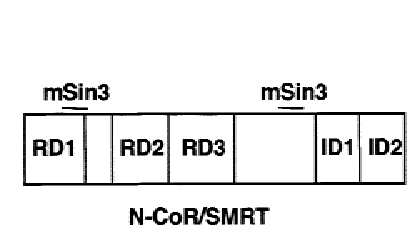

e

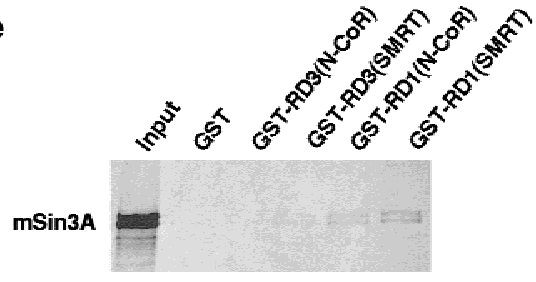

b

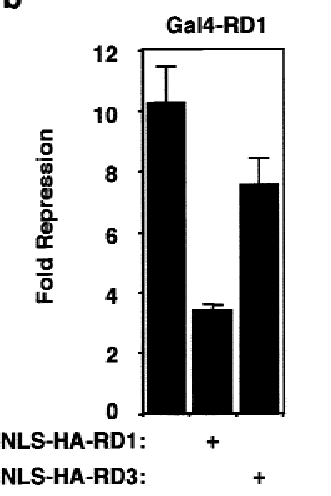

C

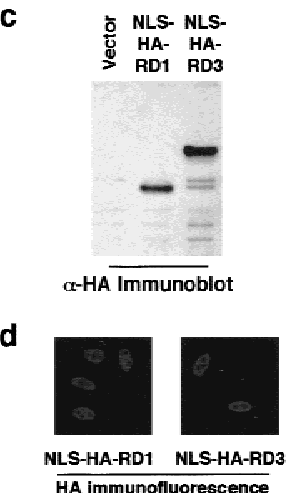

f

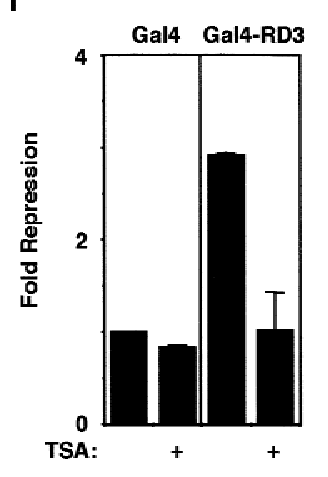

Figure 2. N-CoR and SMRT possess separable and distinct repression domains that operate through independent mechanisms. (a) Schematic representation of N-CoR and SMRT showing the three designated RDs. Interaction domains (ID) are located at the carboxyl terminus and mediate interaction with nuclear hormone receptors. Strong mSin3-binding sites are indicated (Horlein et al. 1995; Alland et al. 1997). (b) Activity of a luciferase reporter in $293 \mathrm{~T}$ cells transfected with Gal4-RD1 (N-CoR 1-312) alone or in the presence of RD1 fused to a nuclear localization signal (NLS-HA-RD1) or NLS-HA-RD3 (SMRT 1031-1505). (c) Immunoblot of whole-cell lysates from 293 T cells transfected with NLS-HA-RD1 and NLS-HA-RD3. (d) Confocal microscopic examination of HeLa cells transfected with NLS-HA-RD1 and NLS-HARD3, stained with anti-HA antibody and Texas Red-conjugated secondary antibody, and visualized by immunofluorescence. (e) Pulldown of $\left[{ }^{35} \mathrm{~S}\right]$ Met-labeled in vitrotranslated $\mathrm{mSin} 3 \mathrm{~A}$ by GST alone or GST$\mathrm{RD}$ fusion proteins as indicated. Input, 10\%. (f) Gal4-DBD and Gal4-SMRT RD3 were transfected and luciferase activity was measured in the absence or presence of 330 nм TSA.
mSin3A/HDAC1, the association of N-CoR with HDAC activity, and the effectiveness of TSA in blocking the mSin3-independent repression by RD3 led us to question whether HDAC1, a class I HDAC, was solely responsible for the deacetylase activity observed in N-CoR immunoprecipitates. The recently identified class II HDACs are more similar to yeast Hdal than Rpd3, and all possess an amino terminal sequence not found in class I HDACs (Fischle et al. 1999; Grozinger et al. 1999; Miska et al. 1999; Verdel and Khochbin 1999|. We hypothesized that endogenous N-CoR might associate with a member of this family. To address this, myc-tagged HDAC4 was overexpressed in HeLa cells. The interaction between N-CoR and HDAC4 was observed both by immunoprecipitation (IP) of N-CoR followed by HDAC4 immunoblot, as well as by myc IP followed by N-CoR immunoblot (Fig. 3a). Similar results were obtained in 293T cells (Fig. 3b).
Class II HDACs directly interact with the mSin3-independent repression domain

The results thus far support a model in which the limiting factor in RD3 function is a class II HDAC, analogous to the recruitment of class I HDACs to RD1 through $\mathrm{mSin} 3 \mathrm{~A}$. To understand the protein interactions involved in RD3 function, we screened a 17-day-old mouse embryo cDNA library in a yeast two-hybrid system using SMRT RD3 as bait. Remarkably, mHDA1 (Miska et al. 1999; Verdel and Khochbin 1999), the mouse homolog of human HDAC5, emerged from this unbiased screen as a strongly interacting protein (Fig. 4a). HDAC5 interacted directly with both N-CoR RD3 and SMRT RD3, but did not interact with N-CoR RD1 in vitro (Fig. 4b). Both HDAC4 and HDAC5 are class II HDACs with high sequence identity, especially in the
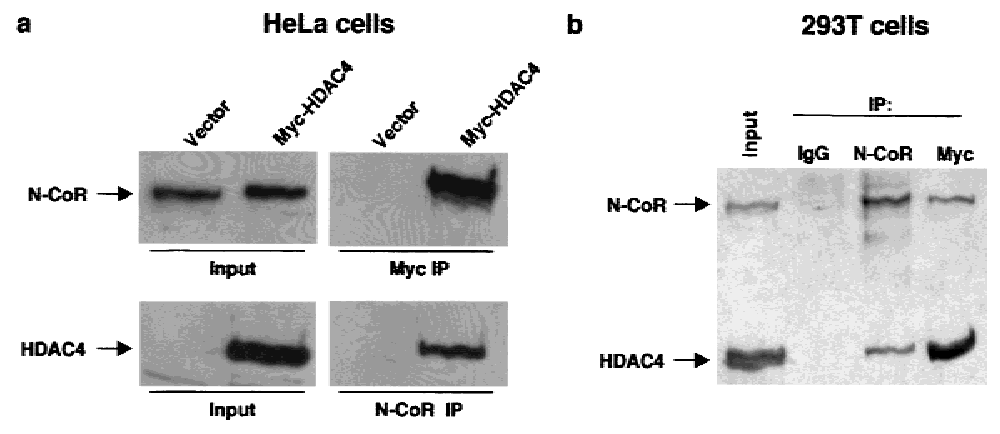

Figure 3. N-CoR interacts with HDAC4 in vivo. (a) $\mathrm{HeLa}$ cells were transfected with empty vector or myctagged HDAC4 (Myc-HDAC4). Whole-cell lysates were immunoprecipitated with myc antibody (top right) or N-CoR antibody (bottom right) and separated by SDS-PAGE. (Top) Cells were immunoblotted with N-CoR antibody; (bottom) with myc antibody. (Left) Inputs to the corresponding lanes at right. (b) 293T cells were transfected with myc-HDAC4 and immunoprecipitated with IgG, myc antibody, or N-CoR antibody, as indicated. Inputs $(5 \%)$ are shown. Samples were immunoblotted for $\mathrm{N}-\mathrm{CoR}$ and myc epitope. 
Figure 4. Repression domain 3 interacts with HDAC4 and HDAC5 in vitro and in vivo. (a) Yeast two-hybrid analysis of mouse HDAC5 clone interaction with Gal4-SMRT RD3. Yeast were transformed with the indicated plasmids and growth was assayed on triple dropout (-His, -Leu, -Trp) plates. The positive control is p53 bait and SV40 Large T antigen prey. (b) $\left[{ }^{35} \mathrm{~S}\right]$ Methionine-labeled in vitrotranslated myc-HDAC1, myc-HDAC4, and VP16HDAC5 were incubated with indicated GST fusion proteins. Inputs, $10 \%$. (c) Mammalian two-hybrid analysis of HDAC5 with repression domains. 293T cells were transfected with Gal4-DBD, Gal4-NCoR RD1, or Gal4-SMRT RD3 in the absence or presence of VP16-HDAC5 and cell lysates were measured for activity of a luciferase reporter. $(d)$ Mammalian two-hybrid analysis as in $c$ except Gal4-N-CoR RD3 was used. (e) Mammalian twohybrid analysis as above with Gal4-DBD, Gal4HDAC1, Gal4-HDAC4, or Gal4-HDAC5 in the presence of vector, VP16-N-CoR RD1, or VP16SMRT RD3. Means \pm S.E.M. are shown.

catalytic domain (Miska et al. 1999). HDAC4 also interacted strongly with both N-CoR RD3 and SMRT RD3 in vitro (Fig. 4b). These interactions were specific for class II HDACs as HDAC1 did not interact with either RD3 or RD1 (Fig. 4b). Mammalian two-hybrid analysis revealed strong interactions between VP16-HDAC5 and Gal4SMRT RD3 (Fig. 4c) and N-CoR RD3 (Fig. 4d) in intact mammalian 293T cells. Reciprocally, VP16-SMRT RD3 and Gal4-HDAC5 also interacted in 293T cells (Fig. 4e). Similar results were obtained with HDAC4 (Fig. 4e). The specificity of these interactions was confirmed by the lack of similar association between HDAC4 and HDAC5 with RD1 (Figs. 4c,d,e) or between HDAC1 and SMRT RD3 (Fig. 4e).

The class II HDAC catalytic domain is required for interaction with corepressor

The carboxyl terminus of HDAC5, containing its catalytic domain, was the region identified as an RD3-interacting protein in the yeast two-hybrid screen. The carboxyl terminus of HDAC4 was similarly found to interact with RD3 in vitro (Fig. 5a). Point mutations that abolish HDAC activity abrogated interaction with RD3 (Fig. 5a). These mutated residues are conserved in

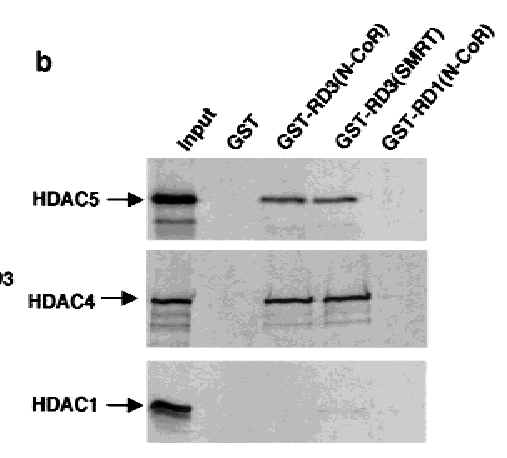

d
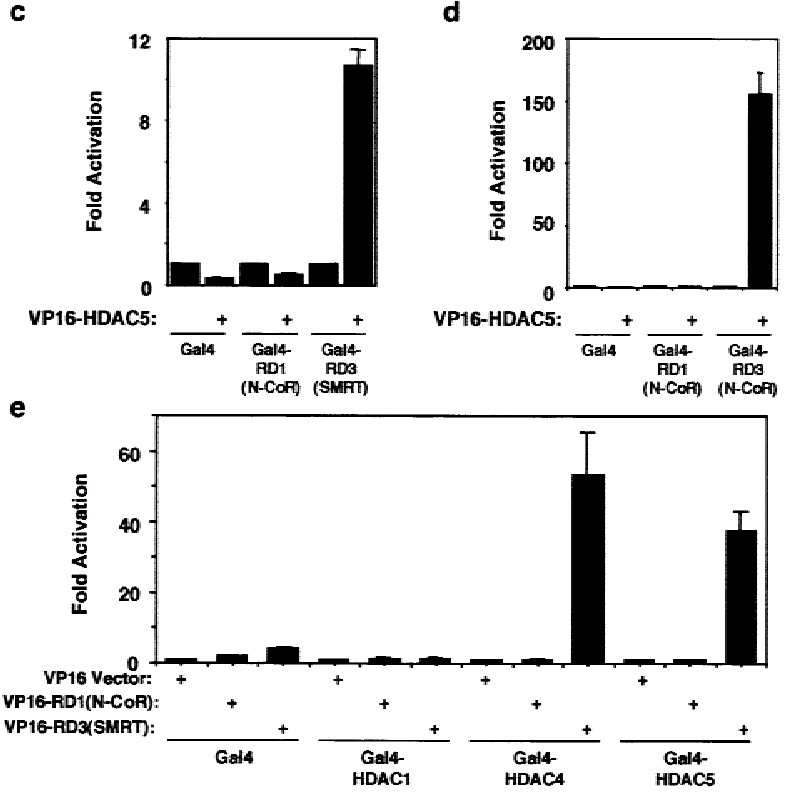

HDAC1, in which the identical substitutions abrogate enzyme activity and, in the case of the D840N, but not the H803A mutation, mSin3A interaction (Hassig et al 1998). HDAC4 and the mutants were myc epitopetagged and expressed in $293 \mathrm{~T}$ cells. The proteins were expressed at similar levels, but only wild-type HDAC4 coimmunoprecipitated endogenous N-CoR (Fig. 5b). Thus, in vivo interactions between HDAC4 and N-CoR corresponded exactly with the in vitro interactions between HDAC4 and SMRT RD3. As expected, only the wild-type HDAC4 possessed HDAC activity (Fig. 5c). Inhibition of wild-type HDAC4 enzyme activity with TSA did not block interaction with N-CoR (Fig. 5d), suggesting that the lack of HDAC activity of the mutants was not solely responsible for their failure to interact with $\mathrm{N}-\mathrm{CoR}$. Furthermore, addition of N-CoR RD3 did not inhibit HDAC4 deacetylase activity in vitro (data not shown).

The repression function of SMRT RD3 sublocalizes to the class II HDAC interaction domain

The HDAC5 interaction domain was sublocalized to the carboxyl terminus of SMRT RD3 (amino acids 1281-1505) (Fig. 6a). HDAC4 interactions localized to 
a

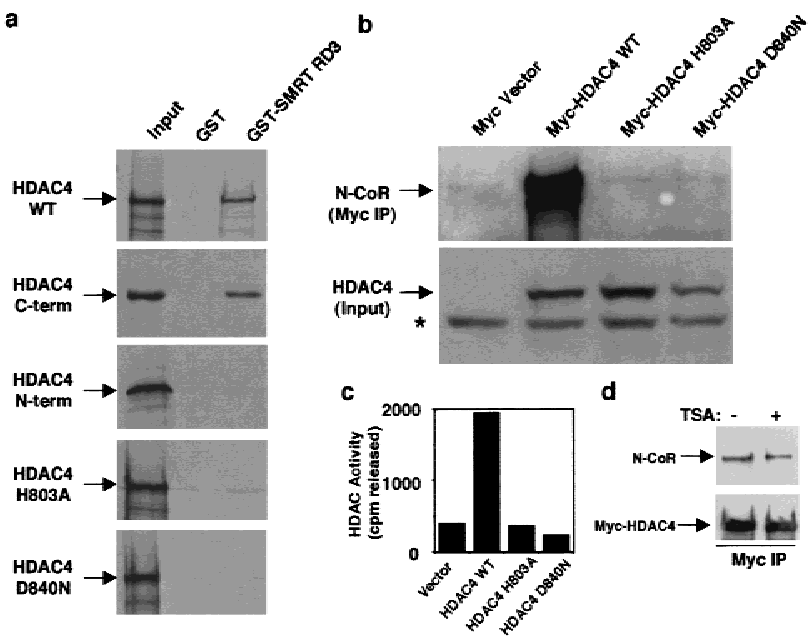

Figure 5. The HDAC4 catalytic domain is required for corepressor interaction. (a) GST or GST-SMRT RD3 were incubated with the indicated $\left[{ }^{35} \mathrm{~S}\right]$ methionine-labeled in vitro-translated proteins in GST pulldown assays. Input, 20\% except for wildtype-HDAC4, $10 \%$. (b) $293 \mathrm{~T}$ cells were transfected with vector or the myc-tagged HDAC4 wild type, H803A mutant, or D840N mutant. Whole-cell lysates were immunoprecipitated with myc antibody and samples were separated by SDS-PAGE and immunoblotted with $\mathrm{N}-\mathrm{CoR}$ antibody (top). Proteins were expressed at equal levels as shown by the myc immunoblot (bottom). The asterisk indicates a background protein. (c) Effects of mutations on HDAC activity. 293T cells were transfected as in $b$, and myc immunoprecipitates were directly assayed for deacetylase activity and are displayed as counts per minute of ${ }^{3} \mathrm{H}$-labeled acetic acid released. $(d)$ TSA has no effect on HDAC4 interaction with $\mathrm{N}$-CoR. 293T cells were transfected with myc-tagged HDAC4 and whole-cell lysates were immunoprecipitated with myc antibody in the absence or presence of $300 \mathrm{nM}$ TSA and immunoblotted with N-CoR or myc antibody.

the same region of RD3, whereas the amino terminus of SMRT RD3 (amino acids 1031-1288) did not interact with either HDAC4 or HDAC5 in vitro (Fig. 6a). This interaction correlated with the repression function of SMRT RD3, which also mapped to the carboxyl terminus (Fig. 6b). These data support the hypothesis that the $\mathrm{RD} 3$ repression function is related to its ability to recruit class II HDACs.

HDAC4 interacts with endogenous N-CoR and SMRT in complexes that do not contain $\mathrm{mSin} 3 A$

We next determined whether mSin3A was a component of HDAC4-N-CoR complexes in intact cells, and whether overexpression of HDAC1 could influence these complexes. HDAC4 and HDAC1 were overexpressed at similar levels in HeLa cells (Fig. 7a). Immunoprecipitates of myc-HDAC4 from transfected cells contained abundant amounts of endogenous N-CoR (Fig. 7b). However, little if any, $\mathrm{mSin} 3 \mathrm{~A}$ was detected in these complexes (Fig. 7b). In addition, endogenous N-CoR did not coimmunoprecipitate with myc-tagged HDAC1, whereas abundant $\mathrm{mSin} 3 \mathrm{~A}$ was present in the HDAC1 complexes (Fig. 7b). These results were confirmed in the reciprocal direction, that is, by immunoprecipitating endogenous N-CoR following transfection of HDAC4 or HDAC1. Only HDAC4 coimmunoprecipitates with NCoR (Fig. 7c). Very similar observations were made for SMRT (Fig. 7d), and all results were repeated in $293 \mathrm{~T}$ cells (data not shown). Finally, we examined interaction of endogenous HDAC4 with endogenous N-CoR and SMRT. Endogenous HDAC4 was detected in immunoprecipitates of endogenous N-CoR and, reciprocally, endogenous N-CoR was associated with immunoisolated endogenous HDAC4 (Fig. 7e). Comparison with the total amount of immunoprecipitated proteins indicated that only a fraction of the N-CoR was in stable HDAC4 complexes, and vice versa. Endogenous SMRT was also detected in HDAC4 immunoprecipitates (Fig. 7f). These results indicate the existence of novel endogenous nuclear receptor corepressor-HDAC4 complexes that largely exclude HDAC1 and mSin3A.

\section{Discussion}

Our results indicate the existence of a novel nuclear receptor corepressor-HDAC complex. Interactions between the nuclear receptor corepressors and HDAC4/ HDAC5 were evident in multiple assay systems, including yeast and GST-pulldown assays, suggesting that the association is direct. The interaction localizes to RD3, whose repression function is TSA sensitive like those of HDAC4 and HDAC5 (data not shown). Furthermore,

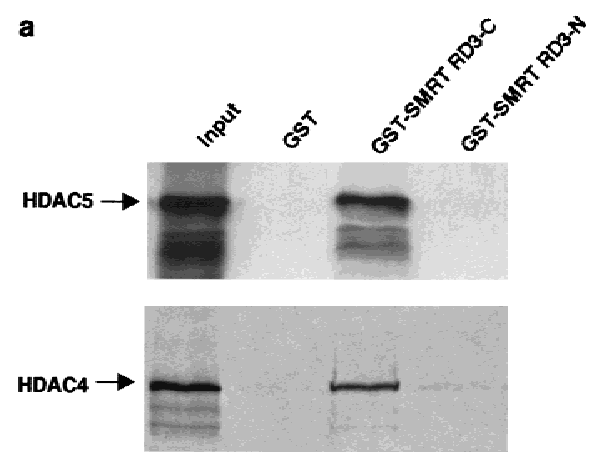

b

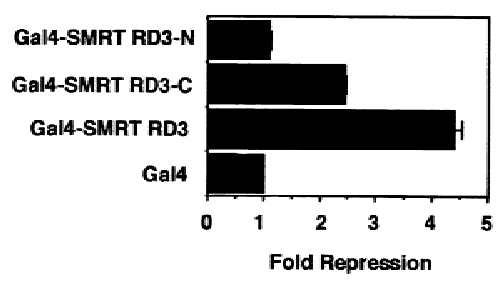

Figure 6. The repression function of RD3 sublocalizes to the class II HDAC interaction domain. (a) $\left[{ }^{35} \mathrm{~S}\right]$ methionine-labeled in vitro-translated HDAC5 and HDAC4 were incubated with GST, GST-SMRT RD3 carboxyl terminus, or GST-SMRT RD3 amino terminus. Input, 10\%. (b) Gal4-SMRT RD3 carboxyl terminus and Gal4-SMRT RD3-amino terminus were transfected into $293 \mathrm{~T}$ cells and luciferase activity was measured. 
Figure 7. N-CoR and SMRT each form complexs with HDAC4 that do not include mSin3A. $(a-c)$ HeLa cells were transfected with empty vector, myc-HDAC4, or myc-HDAC1. (Top) Cells were probed with N-CoR antibody; (middle) cells are probed with mSin3A antibody; (bottom) cells are probed with myc antibody. $(a)$ Immunoblots of inputs. (b) Whole-cell lysates were immunoprecipitated with myc antibody before being immunoblotted as in $a$. (c) Whole-cell lysates of myc-HDAC4- or myc-HDAC1-transfected HeLa cells were immunoprecipitated with IgG or N-CoR IgG before being immunoblotted. (d) Immunoprecipitations were performed as in $b$ and immunoblotted for SMRT (top), mSin3A (middle), and myc epitope (bottom). Inputs are shown in left panels. (e) Whole-cell lysates from untransfected 293T cells immunoprecipitated with either IgG, anti-N-CoR IgG, or anti-HDAC4 antiserum. Samples were immunoblotted for $\mathrm{N}-\mathrm{CoR}$ or HDAC4. Inputs shown, $2 \%$. (f) Same as $e$ except immunoprecipitations were performed with IgG or anti-HDAC4 antiserum, and immunoblotted with SMRT or HDAC4 antibody.
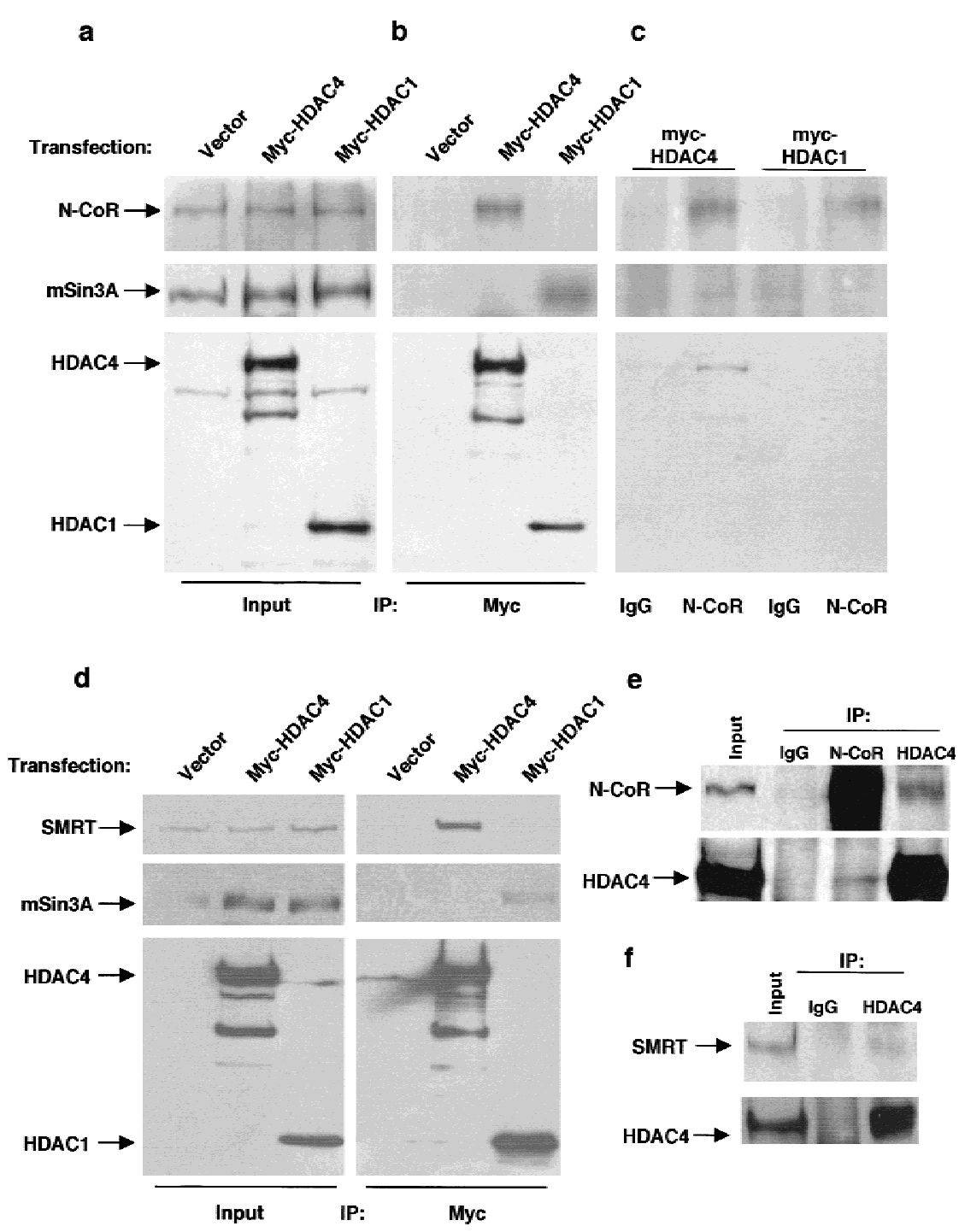

this repression function localizes to the carboxyl terminus of RD3, which is the region that interacts with HDAC4 and HDAC5. Together, these data strongly suggest that the association with HDAC4/HDAC5 is responsible for the TSA-suppressible repression function of RD3.

The N-CoR/SMRT-HDAC4/HDAC5 complex contrasts with the previously described N-CoR/SMRTmSin3-HDAC1 (or HDAC2) complex. RD3 does not interact with $\mathrm{HDAC} 1$ or $\mathrm{mSin} 3 \mathrm{~A}$, whereas RD1 does interact with $\mathrm{mSin} 3 \mathrm{~A}$ in vitro. Thus, the N-CoRassociated HDAC activity may be accounted for by both class I and II HDACs, which associate with corepressor via different mechanisms. As detailed in this report, we have been unable to consistently demonstrate the existence of the $\mathrm{N}-\mathrm{CoR} / \mathrm{mSin} 3 / \mathrm{HDAC} 1$ complex in HeLa and $293 \mathrm{~T}$ cells. Thus, we suggest that this complex is not the sole contributor to the robust N-CoR-associated HDAC activity. This conclusion is surprising in light of the prevailing models for nuclear receptor-mediated repression, but is consistent with the inability of others to detect nuclear receptor corepressors in HDAC1 or Sin3containing complexes (Hassig et al. 1997; Tong et al. 1998; Wade et al. 1998; Zhang et al. 1998b,c).

Although HDAC4 and HDAC5 contain a unique amino terminus that is absent in $\mathrm{HDAC} 1$, the corepressor interactions mapped to the carboxyl terminus of HDAC4 and HDAC5, which contains the HDAC enzyme activity. Several amino acids that are required for catalytic activity of HDAC4 are conserved in HDAC1, including H141 and D176 (Hassig et al. 1998). Mutation of the aspartate residue in HDAC1 also abolished interaction with $\mathrm{mSin} 3 \mathrm{~A}$ and $\mathrm{RbAp} 48$, suggesting a major role in the tertiary structure of the protein, although mutation of the histidine residue did not disrupt protein interactions, suggesting a more specific role in the enzyme activity of HDAC1 (Hassig et al. 1998). Interestingly, both of these mutations in HDAC4 prevent interaction with corepressor. This explains why both of these enzyme-dead mutants of HDAC4 do not function as dominant-negative regulators of corepressor function (E.Y. Huang and M.A. Lazar, unpubl.). We considered the 
possibility that N-CoR may bind to the active site of HDAC4. However, TSA did not prevent the interaction (Fig. 5d), and N-CoR RD3 did not inhibit HDAC4 enzymatic activity, whereas histones did (E.Y. Huang and M.A. Lazar, unpubl.).

The novel corepressor-HDAC4/HDAC5 complex demonstrates that transcriptional repression by nuclear receptors may be functionally executed through different combinations of proteins that possess similar enzymatic activity. This emphasizes the balance between acetylation and deacetylation as reversible determinants of transcriptional activity. Transcriptional activation is mediated by large complexes containing multiple HATs whose function may be activator specific and regulated by cellular signaling events (Korzus et al. 1998; Xu et al. 1998). It is possible that under some circumstances, a single nuclear receptor corepressor complex may recruit multiple HDACs, just as nuclear receptor activation complexes can contain multiple HATs including CBP and the p160 class of coactivators. Although the deacetylase activity and the sensitivity to TSA of HDAC1 and HDAC4 are similar (data not shown; Grozinger et al. 1999; Miska et al. 1999|, the molecular targets of the HDACs may be critical. In addition to histones, protein acetylation regulates a variety of transcriptional regulators (Kouzarides 1999), including both sequence-specific transcription factors p53 (Gu and Roeder 1997; Liu et al. 1999) and GATA-1 (Boyes et al. 1998; Hung et al. 1999), the nuclear receptor coactivator ACTR (Chen et al. 1999), and basal factors such as TFIIF (Imhof et al. 1997). Regulated deacetylation is likely to impact these factors as well. Other proteins in the nuclear receptor corepressor complexes might modify the activity of specific HDACs, as recently described for MTA2 in HDAC1 complexes (Zhang et al. 1999).

We have shown that nuclear receptor corepressors utilize different, noncompeting mechanisms to interact with multiple HDACs. With our present observations, four of the six known mammalian HDACs have been implicated in nuclear hormone receptor repression complexes (Fig. 8). In vivo, the corepressors might, at times,

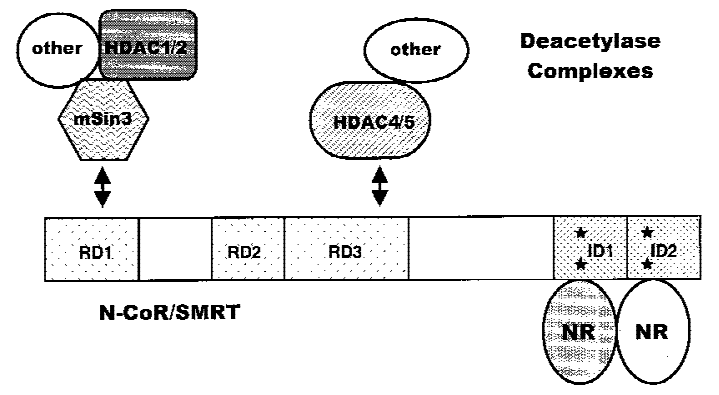

Figure 8. Model of nuclear receptor corepressor-deacetylase interactions. HDAC1/HDAC2 bound to $\mathrm{mSin} 3$ can interact with corepressor via RD1 or a sequence amino-terminal to interaction domain 1 (ID1). In contrast, HDAC4/HDAC5 binds at $\mathrm{RD} 3$ without $\mathrm{mSin} 3$ as a mediator. Other proteins include RbAp46 and RbAp48, and likely differ for HDAC1 and HDAC4 complexes. associate with different HDACs simultaneously, and at other times exist in distinct complexes. The HDACs are expressed in different tissues (Grozinger et al. 1999) and their expression may be regulated during differentiation and by subcellular distribution (Miska et al. 1999; Verdel and Khochbin 1999). Thus, different nuclear receptor corepressor-HDAC complexes may predominate as a function of cell type, cell cycle, or differentiation state. Aberrant recruitment of N-CoR and SMRT is pathogenic in multiple forms of acute myeloid leukemia, and HDAC inhibition has been successfully used to treat human APL (Warrell et al. 1998). The present work suggests that more rational and specific therapies might target a distinct HDAC complex(es) involved in leukemogenesis.

\section{Materials and methods}

Plasmid constructs

The yeast pGAD10-mHDA1 clone was subcloned into pCMXGal4 DBD (Zamir et al. 1997) and pGEX-4T1 (Pharmacia) with EcoRI. Gal4-SMRT RD3, Gal4-NCoR RD1, GST-SMRT RD3, and GST-N-CoR RD1 were described previously (Gelmetti et al. 1998; Zhang et al. 1998a). Gal4-N-CoR RD3 and GST-NCoR RD3 contain amino acids 1017-1506 of N-CoR. HDAC1 was cloned into pCMX-Gal4 DBD to create Gal4-HDAC1. NLS-HA-RD1 and NLS-HA-RD3 were created by taking the EcoRI/NotI fragment from the Gal4 constructs and cloning into pCMV with in-frame HA epitope and nuclear localization signal. pCMX-VP16-HDAC5, -N-CoR RD1, and -SMRT RD3 were created by inserting the NLS-VP16 polylinker from pVP16 into pCMX (Clontech), and subcloning the relevant polypeptides. GST-SMRT RD3-amino terminus (amino acids 1031-1288) and GST-SMRT RD3-carboxyl terminus (amino acids 1281-1505) were cloned by PCR into pGEX-4T1 (Pharmacia). GST-SMRT RD1 contains amino acids 1-303 of full-length SMRT. MycHDAC4 wild-type, H803A, D840N, HDAC4-amino terminus (amino acids 118-611), HDAC4 carboxyl terminus (amino acids 612-1084) as well as myc-HDAC1 were described previously (Miska et al. 1999). HDAC4 carboxyl terminus was also subcloned into pCMX-Gal4 DBD.

\section{Cell culture and transfection}

293 T cells and HeLa cells were maintained in DMEM (high glucose) supplemented with $10 \%$ FBS and L-glutamine (all GIBCO-BRL). Cells were grown at $37^{\circ} \mathrm{C}$ in $5 \% \mathrm{CO}_{2}$. $293 \mathrm{~T}$ cells were transfected by use of the calcium phosphate precipitation technique. HeLa cells were transfected with FuGene 6 (Boehringer Mannheim) according to the manufacturer's instructions. Cells were washed 16-20 hr after transfection and maintained another $24 \mathrm{hr}$ before harvesting for luciferase reporter assays and immunoprecipitations. In luciferase assays, the Gal4 UAS $\mathrm{x}$ 5-SV40-luciferase reporter contains five copies of the Gal4 17mer binding site. TSA (Biomol) was used at 330 nM. For TSA inhibition experiments, 1-day post transfection fresh medium with TSA was added (day 2), and again the following day (day 3), and cells were harvested on day 4. Light units were normalized to a cotransfected $\beta$-galactosidase expression plasmid. Fold repression and fold activation are relative to the Gal4-DBD, and results of duplicate samples are plotted. For immunofluorescence, HeLa cells were grown and transfected on chamber slides (Nalge Nunc). Cells were fixed in $4 \%$ paraformaldehyde, permeabilized in $0.2 \%$ Triton X-100, and blocked with $1 \%$ BSA in 
PBS prior to staining with anti-HA antibody at 1:200 dilution and Texas Red-conjugated goat anti-rabbit IgG secondary antibody (Southern Biotechnology Associates) at 1:75 dilution. Confocal microscropy was performed with a Bio-Rad 1024-ES system on a Nikon Eclipse E800 microscope equipped with a 60x Plan-APO 1.4 NA objective.

\section{Yeast two-hybrid screen}

SMRT amino acids 1031-1505 was used as bait to screen a 17day mouse embryo library (Clontech) as described previously (Zamir et al. 1997). Amino acids 468-991 of mHDAl (Miska et al. 1999; Verdel and Khochbin 1999) (GenBank accession no. AF006602) were isolated from the screen and used in all subsequent assays. This corresponds to human HDAC5 (Grozinger et al. 1999) (GenBank accession no. AF132608) starting at amino acid 601 .

\section{In vitro interaction assays}

Glutathione $S$-transferase (GST) assays were performed as described previously (Zhang et al. 1997). Briefly, GST fusion proteins bound to glutathione beads were incubated with $\left[{ }^{35} \mathrm{~S}\right] \mathrm{me}$ thionine-labeled in vitro-translated proteins at $4^{\circ} \mathrm{C}$, washed, boiled in SDS loading buffer, subjected to SDS-PAGE, and visualized by autoradiography. GST fusion proteins were stained with Coomassie blue to ensure equal loading.

\section{Immunoprecipitations}

Cells were washed in PBS and lysed with NET-N buffer $(150 \mathrm{~mm}$ $\mathrm{NaCl}, 1 \mathrm{~mm}$ EDTA, $20 \mathrm{~mm}$ Tris- $\mathrm{HCl}$ at $\mathrm{pH}$ 8.0, 0.5\% NP-40, and $10 \%$ glycerol) containing protease inhibitor cocktail (Boehringer Mannheim) on ice for $30 \mathrm{~min}$. Lysates were clarified by centrifugation at $12,000 \mathrm{~g}$ for $10 \mathrm{~min}$ at $4^{\circ} \mathrm{C}$. Immunoprecipitations were carried out with mixing at $4^{\circ} \mathrm{C}$ for $2 \mathrm{hr}$ with antibody, then a slurry of protein G-agarose (GIBCO-BRL) was added and rotation continued for another $2 \mathrm{hr}$. Beads were washed four times with NET-N and boiled in $1 \times$ SDS loading buffer.

\section{Immunoblot analysis}

Proteins were separated by SDS-PAGE and transferred to PVDF membranes with HMW transfer buffer $(50 \mathrm{~mm}$ Tris, $380 \mathrm{~mm}$ glycine, $0.1 \%$ SDS, and $20 \%$ methanol). Blots were probed with primary antibodies in TBS containing $0.15 \%$ Tween- 20 and $1 \%$ BSA, followed by horseradish peroxidase-conjugated anti-rabbit or anti-mouse antibody (Boehringer Mannheim) at 1:5000 and ECL reagent (Amersham). Anti-N-CoR antibodies were raised in rabbits against N-CoR amino acids 1944-2453, IgG purified on a protein G column (Pierce), and used at 1:2000 dilution. Anti-SMRT mouse monoclonal antibodies were raised against GST-SMRT amino acids 2004-2310. Anti-mSin3A K-20 rabbit polyclonal (Santa Cruz) was diluted 1:200. Anti-HDAC1 rabbit polyclonal (UBI) was diluted 1:333. Anti-myc 9E10 mouse monoclonal (Santa Cruz) was diluted 1:500. Anti-HDAC4 antibodies were raised in rabbits against HDAC4 amino acids 10671084 and were diluted 1:1000. No cross-reactivity with HDAC5 or HDAC1 could be detected. Anti-HA Y-11 rabbit polyclonal antibody (Santa Cruz) was used at a dilution of 1:500.

\section{HDAC assays}

${ }^{3} \mathrm{H}$-Labeled acetylated HeLa histones were prepared essentially as described (Carmen et al. 1996). Deacetylase activity of im- munoprecipitations was assayed by incubating the pelleted beads with $40,000 \mathrm{cpm}$ of ${ }^{3} \mathrm{H}$-labeled acetylated HeLa histones in a total volume of $200 \mu \mathrm{l}$ of HD buffer $(20 \mathrm{~mm}$ Tris-HCL at $\mathrm{pH}$ 8.0, $150 \mathrm{~mm} \mathrm{NaCl}, 10 \%$ glycerol) at $37^{\circ} \mathrm{C}$ for $2 \mathrm{hr}$. To stop each reaction, $50 \mu \mathrm{l}$ of Stop solution ( $1 \mathrm{M} \mathrm{HCl}, 0.16 \mathrm{M} \mathrm{HAc})$ was added and released ${ }^{3} \mathrm{H}$-labeled acetic acid was extracted with $600 \mu \mathrm{l}$ of ethyl acetate and measured.

\section{Acknowledgments}

The help of the Wistar Institute monoclonal antibody core is gratefully acknowledged. This work was supported by NIH grants DK43806 and DK45586 (M.A.L.) and by the Cancer Research Campaign (to T.K.). DNA sequencing was performed by the University of Pennsylvania sequencing facility, supported in part by the University of Pennsylvania Center for the Molecular Study of Digestive Diseases (P30 DK50306). Confocal microscopy was performed at the Biomedical Imaging Core Laboratories of the University of Pennsylvania, supported in part by the Penn Diabetes and Endocrinology Research Center (5-P30DK19525).

The publication costs of this article were defrayed in part by payment of page charges. This article must therefore be hereby marked "advertisement" in accordance with 18 USC section 1734 solely to indicate this fact.

\section{References}

Alland, L., R. Muhle, H. Hou, J. Potes, L. Chin, N. SchreiberAgus, and R.A. DePinho. 1997. Role for N-CoR and histone deacetylase in Sin3-mediated transcriptional repression. $\mathrm{Na}$ ture 387: 49-55.

Ayer, D.E., Q.A. Lawrence, and R.N. Eisenman. 1995. Mad-Max transcriptional repression is mediated by ternary complex formation with mammalian homologs of yeast repressor Sin3. Cell 80: 767-776.

Blanco, J.C., S. Minucci, J. Lu, X.J. Yang, K.K. Walker, H. Chen, R.M. Evans, Y. Nakatani, and K. Ozato. 1998. The histone acetylase PCAF is a nuclear receptor coactivator. Genes \& Dev. 12: 1638-1651.

Boyes, J., P. Byfield, Y. Nakatani, and Y. Ogryzko. 1998. Regulation of the activity of the transcription factor GATA-1 by acetylation. Nature 396: 594-598.

Carmen, A.A., S.E. Rundlett, and M. Grunstein. 1996. HDA1 and HDA3 are components of a yeast histone deacetylase (HDA) complex. J. Biol. Chem. 271: 15837-15844.

Chakravarti, D., V.J. LaMorte, N.C. Nelson, T. Nakajima, I.G. Schulman, H. Juguilon, M. Montminy, and R.M. Evans. 1996. Role of CBP/p300 in nuclear receptor signaling. Nature 383: 99-103.

Chen, J.D. and R.M. Evans. 1995. A transcriptional co-repressor that interacts with nuclear hormone receptors. Nature 377: 454-457.

Chen, H., R.J. Lin, W. Xie, D. Wilpitz, and R.M. Evans. 1999. Regulation of hormone-induced histone hyperacetylation and gene activation by acetylation of an acetylase. Cell 98: 675-686.

Fischle, W., S. Emiliani, M.J. Hendzel, T. Nagase, N. Nomura, W. Voelter, and E. Verdin. 1999. A new family of human histone deacetylases related to Saccharomyces cerevisiae HDAlp. J. Biol. Chem. 274: 11713-11720.

Gelmetti, V., J. Zhang, M. Fanelli, S. Minucci, P.G. Pelicci, and M.A. Lazar. 1998. Aberrant recruitment of the nuclear receptor corepressor-histone deacetylase complex by the acute 
myeloid leukemia fusion partner ETO. Mol. Cell. Biol. 18: 7185-7191.

Glass, C.K., D.W. Rose, and M.G. Rosenfeld. 1997. Nuclear receptor coactivators. Curr. Opin. Cell. Biol. 9: 222-232.

Grignani, F., S. DeMatteis, C. Nervi, L. Tomassoni, V. Gelmetti, M. Cioce, M. Fanelli, M. Ruthardt, F.F. Ferrara, I. Zamir et al. 1998. Fusion proteins of the retinoic acid receptor- $\alpha$ recruit histone deacetylase in promyelocytic leukaemia. Nature 391: 815-818.

Grozinger, C.M., C.A. Hassig, and S.L. Schreiber. 1999. Three proteins define a class of human histone deacetylases related to yeast Hdalp. Proc. Natl. Acad. Sci. 96: 4868-4873.

$\mathrm{Gu}$, W. and R.G. Roeder. 1997. Activation of p53 sequencespecific DNA binding by acetylation of the p53 C-terminal domain. Cell 90: 595-606.

Hanstein, B., R. Eckner, J. DiRenzo, S. Halachmi, H. Liu, B. Searcy, R. Kurokawa, and M. Brown. 1996. P300 is a component of an estrogen receptor coactivator complex. Proc. Natl. Acad. Sci. 93: 11540-11545.

Hassig, C.A., T.C. Fleischer, A.N. Billin, S.L. Schreiber, and D.E. Ayer. 1997. Histone deacetylase activity is required for full transcriptional repression by mSin3A. Cell 89: 341-348.

Hassig, C.A., J.K. Tong, T.C. Fleischer, T. Owa, P.G. Grable, D.E. Ayer, and S.L. Schreiber. 1998. A role for histone deacetylase activity in HDAC1-mediated transcriptional repression. Proc. Nat1. Acad. Sci. 95: 3519-3524.

He, L.Z., F. Guidez, C. Tribioli, D. Peruzzi, M. Ruthardt, A. Zelent, and P.P. Pandolfi. 1998. Distinct interactions of PML-RAR $\alpha$ and PLZF-RAR $\alpha$ with co-repressors determine differential responses to RA in APL. Nat. Genet. 18: 126135.

Heinzel, T., R.M. Lavinsky, T.-M. Mullen, M. Soderstrom, C.D. Laherty, J. Torchia, W.-M. Yuang, G. Brard, S.D. Ngo, J.R. Davie et al. 1997. A complex containing $\mathrm{N}-\mathrm{CoR}, \mathrm{mSin} 3$ and histone deacetylase mediates transcriptional repression. $\mathrm{Na}$ ture 387: 43-48.

Hong, S.H., G. David, C.W. Wong, A. Dejean, and M.L. Privalsky. 1997. SMRT corepressor interacts with PLZF and with the PML-retinoic acid receptor $\alpha$ and PLZF-RAR $\alpha$ oncoproteins associated with acute promyelocytic leukemia. Proc. Nat1. Acad. Sci. 94: 9028-9033.

Horlein, A.J., A.M. Naar, T. Heinzel, J. Torchia, B. Gloss, R. Kurokawa, A. Ryan, Y. Kamei, M. Soderstrom, C.K. Glass et al. 1995. Ligand-independent repression by the thyroid hormone receptor mediated by a nuclear receptor co-repressor. Nature 377: 397-404.

Hung, H.L., J. Lau, A.Y. Kim, M.J. Weiss, and G.A. Blobel. 1999. CREB-binding protein acetylates hematopoietic transcription factor GATA-1 at functionally important sites. Mol. Cell. Biol. 19: 3496-3505.

Imhof, A., S.J. Yang, V.V. Ogryzko, Y. Nakatani, A.P. Wolffe, and H. Ge. 1997. Acetylation of general transcription factors by histone acetyltransferases. Curr. Biol. 7: 689-692.

Kamei, Y., L. Xu, T. Heinzel, J. Torchia, R. Kurokawa, B. Gloss, S.-C. Lin, R.A. Heyman, D.W. Rose, C.K. Glass et al. 1996. A CBP integrator complex mediates transcriptional activation and AP-1 inhibition by nuclear receptors. Cell 85: 403-414.

Korzus, E., J. Torchia, D.W. Rose, L. Xu, R. Kurokawa, E.M. McInerney, T.M. Mullen, C.K. Glass, and M.G. Rosenfeld. 1998. Transcription factor-specific requirements for coactivators and their acetyltransferase functions. Science 279: 703-707.

Kouzarides, T. 1999. Histone acetylases and deacetylases in cell proliferation. Curr. Opin. Genet. Dev. 9: 40-48.

Lin, R.J., L. Nagy, S. Inoue, W. Shao, W.H. Miller, and R.M. Evans. 1998. Role of the histone deacetylase complex in acute promyelocytic leukaemia. Nature 391: 811-814.

Liu, L., D.M. Scolnick, R.C. Trievel, H.B. Zhang, R. Marmorstein, T.D. Halazonetis, and S.L. Berger. 1999. p53 sites acetylated in vitro by PCAF and p300 are acetylated in vivo in response to DNA damage. Mol. Cell. Biol. 19: 1202-1209.

Lutterbach, B., J.J. Westendorf, B. Linggi, A. Patten, M. Moniwa, J.R. Davie, K.D. Huynh, V.J. Bardwell, R.M. Lavinsky, M.G. Rosenfeld et al. 1998. ETO, a target of $\mathrm{t}(8 ; 21)$ in acute leukemia, interacts with the N-CoR and $\mathrm{mSin} 3$ corepressors. Mol. Cell. Biol. 18: 7176-7184.

Miska, E.A., C. Karlsson, E. Langley, S.J. Nielsen, J. Pines, and T. Kouzarides. 1999. HDAC4 associates with and represses the MEF2 transcription factor. EMBO J. 18: 5099-5017.

Nagy, L., H.-Y. Kao, D. Chakvarkti, R.J. Lin, C.A. Hassig, D.E. Ayer, S.L. Schreiber, and R.M. Evans. 1997. Nuclear receptor repression mediated by a complex containing SMRT, mSin3A, and histone deacetylase. Cell 89: 373-380.

Ordentlich, P., M. Downes, W. Xie, A. Genin, N.B. Spinner, and R.M. Evans. 1999. Unique forms of human and mouse nuclear receptor corepressor SMRT. Proc. Natl. Acad. Sci. 96: 2639-2644.

Park, E.J., D.J. Schroen, M. Yang, H. Li, L. Li, and J.D. Chen. 1999. SMRTe, a silencing mediator for retinoid and thyroid hormone receptors-extended isoform that is more related to the nuclear receptor corepressor. Proc. Natl. Acad. Sci. 96: 3519-3524.

Pazin, M.J. and J.T. Kadonaga. 1997. What's up and down with histone deacetylation and transcription? Cell 89: 325-328.

Rundlett, S.E., A.A. Carmen, R. Kobayashi, S. Bavykin, B.M. Turner, and M. Grunstein. 1996. HDA1 and RPD3 are members of distinct yeast histone deacetylase complexes that regulate silencing and transcription. Proc. Natl. Acad. Sci. 93: 14503-14508.

Safer, J.D., J.F. Langlois, R. Cohen, T. Monden, D. John-Hope, J. Madura, A.N. Hollenberg, and F.E. Wondisford. 1997. Isoform variable action among thyroid hormone receptor mutants provides insight into pituitary resistance to thyroid hormone. Mol. Endocrinol. 10: 16-26.

Seol, W., M.J. Mahon, Y.-K. Lee, and D.D. Moore. 1996. Two receptor interacting domains in the nuclear hormone receptor corepressor RIP13/N-CoR. Mol. Endocrinol. 10: 16461655.

Struhl, K. 1998. Histone acetylation and transcriptional regulatory mechanisms. Genes \& Dev. 12: 599-606.

Tong, J.K., C.A. Hassig, G.R. Schnitzler, R.E. Kingston, and S.L. Schreiber. 1998. Chromatin deacetylation by an ATP-dependent nucleosome remodelling complex. Nature 395: 917-921.

Verdel, A. and S. Khochbin. 1999. Identification of a new family of higher eukaryotic histone deacetylases. Coordinate expression of differentiation-dependent chromatin modifiers. J. Biol. Chem. 274: 2440-2445.

Wade, P.A., P.L. Jones, D. Vermaak, and A.P. Wolffe. 1998. A multiple subunit Mi-2 histone deacetylase from Xenopus laevis cofractionates with an associated Snf2 superfamily ATPase. Curr. Biol. 8: 843-846.

Wang, J., T. Hoshino, R.L. Redner, S. Kajigaya, and J.M. Liu. 1998. ETO, fusion partner in $\mathrm{t}(8 ; 21)$ acute myeloid leukemia, represses transcription by interaction with the human N-CoR/mSin3/HDAC1 complex. Proc. Natl. Acad. Sci. 95: 10860-10865.

Warrell, R.P., L.Z. He, E. Calleja, and P.P. Pandolfi. 1998. Therapeutic targeting of transcription in acute promyelocytic leukemia by use of an inhibitor of histone deacetylase. J. Natl. Cancer Inst. 90: 1621-1625.

Westin, S., R. Kurokawa, R.T. Nolte, G.B. Wisely, E.M. McInerney, D.W. Rose, M.V. Milburn, M.G. Rosenfeld, and C.K. 
Huang et al.

Glass. 1998. Interactions controlling the assembly of nuclear receptor heterodimers and co-activators. Nature 395: 199202.

Xu, L., R.M. Lavinsky, J.S. Dasen, S.E. Flynn, E.M. McInerney, T.M. Mullen, T. Heinzel, D. Szeto, E. Korzus, R. Durokawa et al. 1998. Signal-specific co-activator domain requirements for Pit-1 activation. Nature 395: 301-306.

Yoh, S.M., V.K.K. Chatterjee, and M.L. Privalsky. 1997. Thyroid hormone resistance syndrome manifests as an aberrant interaction between mutant T3 receptors and transcriptional corepressors. Mol. Endocrinol. 11: 470-480.

Zamir, I., H.P. Harding, G.B. Atkins, A. Horlein, C.K. Glass, M.G. Rosenfeld, and M.A. Lazar. 1996. A nuclear hormone receptor corepressor mediates transcriptional silencing by receptors with different repression domains. Mol. Cell. Biol. 16: 5458-5465.

Zamir, I., J. Zhang, and M.A. Lazar. 1997. Stoichiometric and steric principles govering repression by nuclear hormone receptors. Genes \& Dev. 11: 835-846.

Zhang, J., I. Zamir, and M.A. Lazar. 1997. Differential recognition of liganded and unliganded thyroid hormone receptor by retinoid X receptor regulates transcriptional repression. Mol. Cell. Biol. 17: 6887-6897.

Zhang, J., M.G. Guenther, R.W. Carthew, and M.A. Lazar. 1998a. Proteasomal regulation of nuclear receptor corepressor-mediated repression. Genes \& Dev. 12: 1776-1880.

Zhang, Y., G. LeRoy, H.P. Seelig, W.S. Lane, and D. Reinberg. 1998b. The dermatomyositis-specific autoantigen Mi2 is a component of a complex containing histone deacetylase and nucelosome remodeling activities. Cell 95: 279-289.

Zhang, Y., Z.W. Sun, R. Iratni, B.H. Erdjument, P. Tempst, M. Hampsey, and D. Reinberg. 1998c. SAP30, a novel protein conserved between human and yeast, is a component of a histone deacetylase complex. Mol. Cell. 1: 1021-1031.

Zhang, Y., H.H. Ng, H. Erdjument-Bromage, P. Tempst, A. Bird, and D. Reinberg. 1999. Analysis of the NuRD subunits reveals a histone deacetylase core complex and a connection with DNA methylation. Genes \& Dev. 13: 1924-1935. 


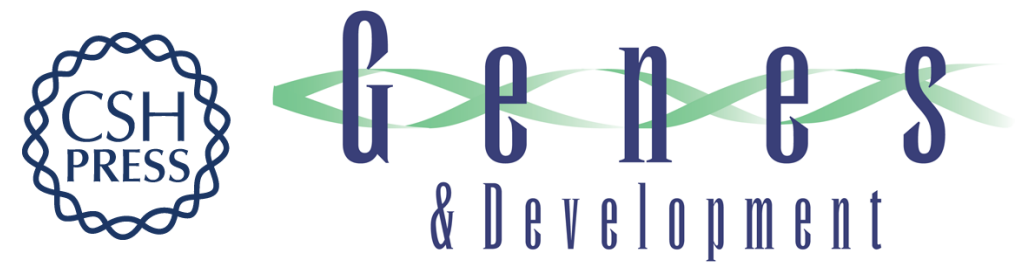

\section{Nuclear receptor corepressors partner with class II histone deacetylases in a Sin3-independent repression pathway}

Eric Y. Huang, Jinsong Zhang, Eric A. Miska, et al.

Genes Dev. 2000, 14:

Access the most recent version at doi:10.1101/gad.14.1.45

$\begin{array}{ll}\text { References } & \begin{array}{l}\text { This article cites } 52 \text { articles, } 23 \text { of which can be accessed free at: } \\ \text { http://genesdev.cshlp.org/content/14/1/45.full.html\#ref-list-1 }\end{array}\end{array}$

License

Email Alerting Receive free email alerts when new articles cite this article - sign up in the box at the top Service right corner of the article or click here.

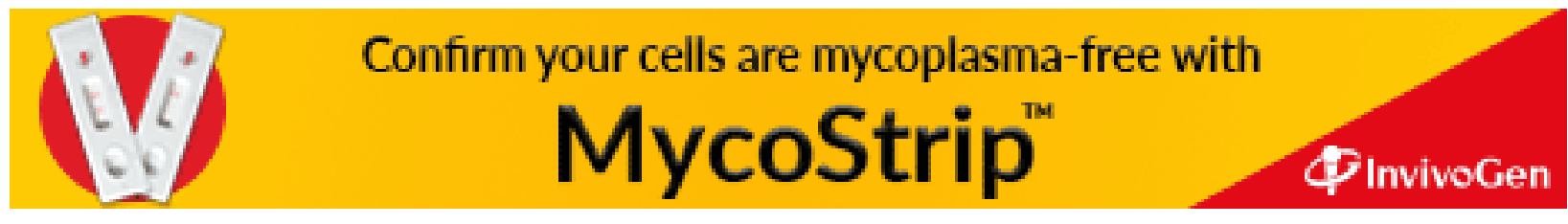

